chitis, all these and other changes proceed from derangements of the nutritive functions.

III. The third class of symptoms are those exemplified in the Gerital FuncTIONS.

The symptoms to which we have heretofore alluded, are almost, without exception, common to both sexes. The third class differ materially in each.

The alterations in the external functions of generation may more properly be considered under the head of exterial appearance. In this place we may consider the functions destined to the reproduction. of the species.

In man the secretion of semen is but rarely augmented in disease. It takes place in erotic melancholy, and is one of the principal symptoms of satyriasmus. The diminution or suspension of the secretion is, on the contrary, a frequent occurrence. The ejaculation of the semen is subject to frequent derangement; it may be painful, feeble, or impracticable. Impotency, or an incapacity to priapism, may also frequently occur from various causes.

The reproductive functions of woman are subject to several maladies. The menstrual Iochia, and lactation, merit particular study. The augmentation, diminution, and suppression of the catamenia, are familiar examples. The lochia, too, may be morbidly increased, diminished, or suppressed. The secretion of milk is never increased by disease, but it is frequently lessened and sometimes suspended. Sterility amongst women may existlike impotence amongst men, and springs from numberless and very different causes.

The venereal appetite may be increased in both sexes, as in satyriasmus and nymphomania, and diminished as in most diseases, and especially in such as affect people given to masturbation. Its entire abolition is called anaphrodisia; and it is often perverted in mania and other neuroses.

Such are the principal symptoms supplied by the generative functions. To these may be added the facts obtained by means of the speculum uteri, such as induration of the os tince, its inequalities, the tumours, ulcers, and fistulx that may exist in the ragina. I must confess, bowever, I think the evil incurred greater than the good derived from this in:trument.

After havino tak en a comprehensive view of the various functions, we shall by a careful collation of the symptoms be enabled without much difficulty to arrive at a satisfactory conclusion as to the nature of the disease. This is diagnosis, which we shall hereafter have to consider in each particu. lar disease; and having arrived at this point, the therapeutic stage will be easy, and the prognosis in most instances unequi vocal.

1 have thus endeavoured, Gentlemen, to give you a faint outline, a kind of bird's-eye view, of the field which we are to survey and cultivate. 'I'he picture you must fill up for yourselves. The acquisition of knowledge is a good which every man must achieve for himself. All attempts, therefore, to im. press knowledge upon the passive mind must be vain. Indeed, I do not pretend to assume the didactic character of instructor; all I aim at is, to induce you to join beartily with me in the cultivation of this interest. ing province of science. We may afford each other important assistance; and I am convinced that if we are sincere and constant in our exertions, we shall succeed in accumulating such a store of well-arranged knowledge as will make us highly useful as members of the community, and will be a source of comfort and consolation to our. selves to the latest moment of existence.

2.

\section{CASE OF FISTULA.}

By Richard Lanyon, Jun. Esq. F.A.S. \&;. Lostwithiel.

I TROUBLE your readers with the follow. ing narration, under the assurance, that notwithstanding much has been written respecting fistula about the rectum, there still remains considerable difficulty in managing these troublesome complaints. Division of parts is not invariably succeeded by the granulatory process, and the practitioner is frequently not a little perplexed in the use of curative means after the bistoury has been freely used. Diseases of this riature, maintained and frequently caused by cachectic habits, present, even in the most slilful hands, no mean obstacles to their removal; every recorded case, therefore, must possess intrinsic value peculiarly its own.

In the month of April, 1828, a young man, who leads rather a debauched life, applied for the relief of fistula in perineo. Several months previously a small abscess bad formed in the perinæum, and a thin ichorous discharge was the consequence. On introducing the probe, an opening was found leading to a superficial sinus, diserg. ing from the gut and running parallel with the integuments, which it was proposed to treat by incision, but was met by obstinate resistance on the part of the patient. At length, however, he yielded, and a longitudinal opening of about an inch and a half exposed another sinus, running almost at right angles with the first, in the direction of the rectum, and so contracted as to ren- 
ler the director and bistoury inadmissible; the nation. The nary, which possesses able it extended about two inches, and was se- medical men could be so arranged as to prrparated by a thin partition from the gut. form all the necessary duties of the existing Guided by the probe, one arm of a pair of quarantine establishments without any inscissors was passed into the sinus, and the crease of its numerical force. The Dutch other carefully introduced into the rectum, haveno distinct establishment of quarantine, and thus the lower or most depending part and they do not appear to be more subject of the fistula was incised; butits upper part to contagious diseases than those counbeing less constricted, the operation was tries who are so amply provided with them. completed by the director and bistoury. It is not only wicked but unphilosophical An oiled tent was introduced every day, in the extreme, to assume contagion as a but the parts did not granulate, nor was cause of disease without the strongest proof there any discharge ; the tent was now omit- of its being so. I am quite certain that ted for several hours each day, but with no epidemical diseases, spreading themselves better result.

$\mathrm{B}_{0}$ Tinct. cantharidis, $3^{\mathrm{i}}$. Aque, ž̉. M. fiat injectio.

Some of this injection was used daily, and a discharge of thick pus followed, succeeded by granulation. After a while the healing process became suspended, and about an inch of the sinus remained open. The occasional use of the nitrate of silver completed the cure of the internal sinus; the external was successfully treated by means of adhesive straps. There was not the least disposition to constipation throughout the disease, and the cure occupied several weeks.

October, 1831.

\section{CHOLERA MORBUS.}

DECTARATION AGAINST THE DOCTHINE THAT THE CHOLERA IS CONTAGIOUS.

The Editor of Tire Lincet is requested to give the following extract fom a letter from Glasgow a pluce in his valuable Jourinal, as it comes from an individual of great profeśsional experience.

A constant Reader.

London, 15th October, 1831.

Extract of a Letier from Glasgow, dated 10th October, 1831.

"My professional duties have placed me in a situation which enabled me to trace yellow fever to its proper source, and also 10 observe the character of cholera morbus in tropical climates, and I have no hesitation in stating, that neither of these disedses originated in a contagious cause. The contagionists in yellow fever and cholera morbus, with their comfrerie, the president and fellows of the College of Jhysicians, may be justly designated the borouyhnongers in medicine, as, by invention and the perversion of facts, they encourage delusions of the most injurious nature, and chus uphold a system of quarantine laws, in the fruits of which they partake, to the great injury of over extensive districts, as it were per saltem, are never contagious, as maladies of the contagious character, in their commence. ment, creep gradually from person to person, and thus diffuse themselves orer the community in which they have been introduced. The rapidity with which yellow fever and cholera extend their influence is utterly at variance with the doctrine of contagion, as founded on truth, analogy, and impartial observation. The number of persons simultaneously attacked with cholera and yellow fever, the marches and counter marches (if I may so express myself) of those diseases, without any evident connecting link or medium calculated to satisfactorily convey them from the point they set out from, to their re. mote destinations, defy all the laws and phenomena of contagious diseases. The changed conditions of the air, and of the emanations from the earth, or a combination of both, will be found to be the causes of these epidemical diseases, and not contagion. The importation of yellow fever into the WestIndies, in the ship Hankey, from Bulam, as traced by the contagionists, is chimerical in the extreme; it is a worthless fable, and proves the authors of it to be utterly incapable of just reasoning or thinking. I am convinced, that if a premium were offered for the importation of cholera morbus, or yellow fever, into this kingdom by means of the dress or bedclothes in which patients had died in the most inveterate forms of these distases, the thing would be found impossible. No mischiet would arise unless the improrters of the bedclothes conld $1 \mathrm{~m}$ port the peculiar constitution of the atmo= sphere and of the earth under which these maladies originated. 'Tlie doctine of contagion, as influencug cholera morbus and yellow fever, ought to be thoroughly invrstigated : such an investigution is loudly called for by the interests of humanity as well as commerce, as the discordant opin. ions on this lead, arising out of an unprincipled disposition to uphold the existing quarantine establishments, are in the highest degree injurious to coinmerce and humanity."

No. 496. 